\title{
Association between RASSF1A Promoter Hypermethylation and Oncogenic HPV Infection Status in Invasive Cervical Cancer: a Meta-analysis
}

\author{
Jin-Yun Li1 ${ }^{1,2}$, Tao Huang ${ }^{1,2}$, Cheng Zhang', Dan-Jie Jiang', Qing-Xiao Hong ${ }^{1}$, \\ Hui-Hui Ji ${ }^{1}$, Meng Ye²*, Shi-Wei Duan ${ }^{1 *}$
}

\begin{abstract}
Cervical carcinoma is the main cause of cancer-related mortality in women and is correlated with more than 15 risk cofactors, including infection of cervical cells with high-risk types of HPV (hrHPV). Indeed, both aberrant methylation of the RASSF 1A promoter and hrHPV infection are often observed in cervical carcinomas. The purpose of our meta-analysis was to evaluate the role of $R A S S F 1 A$ promoter methylation and hrHPV infection in cervical cancer. Our meta-analysis involved 895 cervical cancer patients and 454 control patients from 15 studies. Our results suggested that RASSF1A promoter hypermethylation increased the risk of cervical cancer $\left(\mathrm{OR}=9.77,95 \% \mathrm{CI}=[3.06,31.26], P=0.0001, \mathrm{I}^{2}=78 \%\right)$. By grouping cases according to cancer subtypes, we found that HPV infection was higher in cervical squamous cell carcinomas (SCCs) than in cervical adenocarcinomas/ adenosquamous cancers $(\mathrm{ACs} / \mathrm{ASCs})\left(\mathrm{OR}=4.00,95 \% \mathrm{CI}=[1.41,11.30], P=0.009, \mathrm{I}^{2}=55 \%\right)$. Interestingly, HPV infection tended to occur in cervical cancers with relatively low levels of RASSF1A promoter methylation $\left(\mathrm{OR}=0.59,95 \% \mathrm{CI}=[0.36,0.99], P=0.05, I^{2}=0 \%\right)$. Our study provides evidence of a possible interaction between HPV infection and RASSF 1A promoter methylation in the development of cervical cancers.
\end{abstract}

Keywords: Cervical cancer - RASSF1A methylation - human papilloma virus - HPV infection - meta-analysis

Asian Pac J Cancer Prev, 16 (14), 5749-5754

\section{Introduction}

Cervical cancers remain the third leading cause of genital system cancer-related mortality in women, despite the widespread application of screening methods for the prevention and early detection of cervical cancer. Numerous epidemiological studies have proved that genital HPV infections, as the central etiological factor, were highly correlated with cervical cancer and were present in almost $90 \%$ of cervical carcinomas (Bosch et al., 1995). Among all of the HPV subtypes, of which more than 100 have been identified, only the high-risk HPVs (including types 16, 18, 33, 58 and 59) were strongly associated with the progression of cervical cancers (Munoz et al., 2003). However, a majority of the women infected with HPV do not develop cervical cancer, and $90 \%$ of HPV infections usually resolve on their own within 2 years. Therefore, other complex genetic and epigenetic alterations might cooperate to drive the development of cervical cancers.

In addition to cancer-related genetic mutations (Zhu et al., 2014), epigenetic alterations without changes in gene sequences, such as promoter hypermethylation, are relatively common events in many human cancers (Bird, 1986; Jiang et al., 2014a; Jiang et al., 2014b). Several tumor suppressor genes (TSGs) have been found to be frequently inactivated by promoter hypermethylation and linked with the pathogenesis and progression of cervical cancers (Cheung et al., 2004; Jha et al., 2012). RASSF $1 A$ (Ras association domain family 1 isoform) is one of the RASSF (Ras association domain family) members, which play an extremely important role in various cellular processes such as the modulation of apoptosis, migration and regulation of adhesion (van der Weyden and Adams, 2007; Richter et al., 2009). Aberrant methylation of the RASSF 1A promoter was often observed in cervical carcinomas (Yu et al., 2003; Maliukova et al., 2004).

Meta-analyses provide the advantage of combining data from multiple studies and establishing relationships across studies, allowing one to analyze larger sample numbers and draw relatively more reliable conclusions(Lipsey and Wilson, 2001). In light of previous studies, we performed a meta-analysis of the studies monitoring the RASSF1A promoter methylation status and HPV infection status in cervical cancer and attempted to establish the role of these risk cofactors in cervical cancer development. 


\section{Materials and Methods}

\section{Identification of relevant studies}

All relevant studies, updated until October 8, 2014, were systematically searched from the PubMed, China National Knowledge Infrastructure, and Wanfang databases using the keywords "RASSF1A methylation" and "HPV" or "human papilloma virus" in conjunction with "cervical cancer" or "cervical intraepithelial neoplasia" or "uterine cervix cancer". In addition, we conducted a manual search to identify other potential studies within the reference lists of the retrieved studies.

\section{Inclusion and exclusion criteria}

All selected studies met the following eligibility criteria: (1) the study should refer to the methylation status of RASSF $1 A$ or HPV infection in invasive cervical cancers; (2) the study should assess and present the details of the RASSF $1 A$ methylation status in cervical cancer tissues; and (3) the study should provide detailed information on the HPV infection status, such as the HPV subtype. Studies assessing the RASSF 1A methylation status in cell lines or in cervical intraepithelial neoplasia rather than in invasive cervical cancers were excluded. We defined the case groups as invasive cervical carcinomas, and the control cases were the corresponding normal tissues or benign diseases. Furthermore, studies without detailed information on gene methylation and HPV infection data were removed from our analysis.

\section{Data extraction}

From the eligible studies, we extracted the first author's name, publication year, ethnicity of the study subjects, methylation assessment method, number of cervical cancers and corresponding normal control group, hrHPV infection status as hrHPV-positive and hrHPVnegative cervical cancers, histological type of the tumors and frequency of RASSF $1 A$ promoter methylation.

\section{Statistical analysis}

Using the Review Manager 5 software, we calculated the combined odds ratios (ORs) with corresponding $95 \%$ confidence intervals (95\%CIs) to estimate the associations included in our meta-analysis. An $\mathrm{I}^{2}$ metric analysis was used to assess heterogeneity among the included studies. When the $\mathrm{I}^{2}$ metric value was more than $50 \%$, we considered the involved studies to show an obvious level of heterogeneity that needed to be adjusted by applying a random-effect model. Otherwise, a fixed-effect model was used.

\section{Results}

A total of 677 studies were initially retrieved using the aforementioned keywords (Figure 1). After removing duplications, 590 studies remained for further filtration. After carefully scrutinizing the titles and abstracts of the remaining articles, we removed 278 irrelevant studies, 53 entries that were review articles, abstracts or inaccessible full-text manuscripts, 87 non-human studies, and 160 studies that were not case-controlled or lacked the RASSF $1 A$ methylation data. Along with an additional 3 studies that were manually selected from the reference lists included within the retrieved studies, we collected

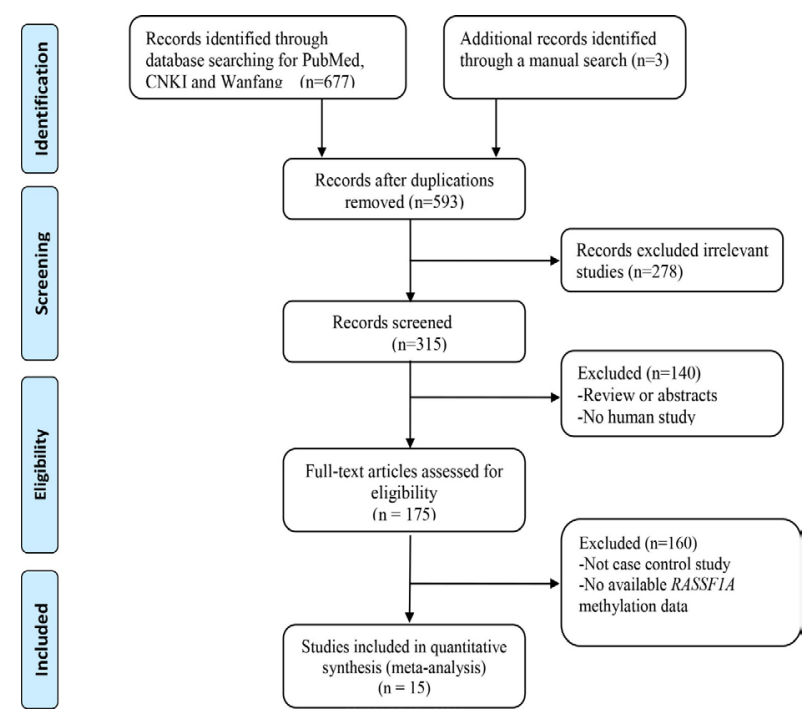

Figure 1. Flow Diagram Representing the Stepwise Selection of Relevant Studies

Odds Ratio

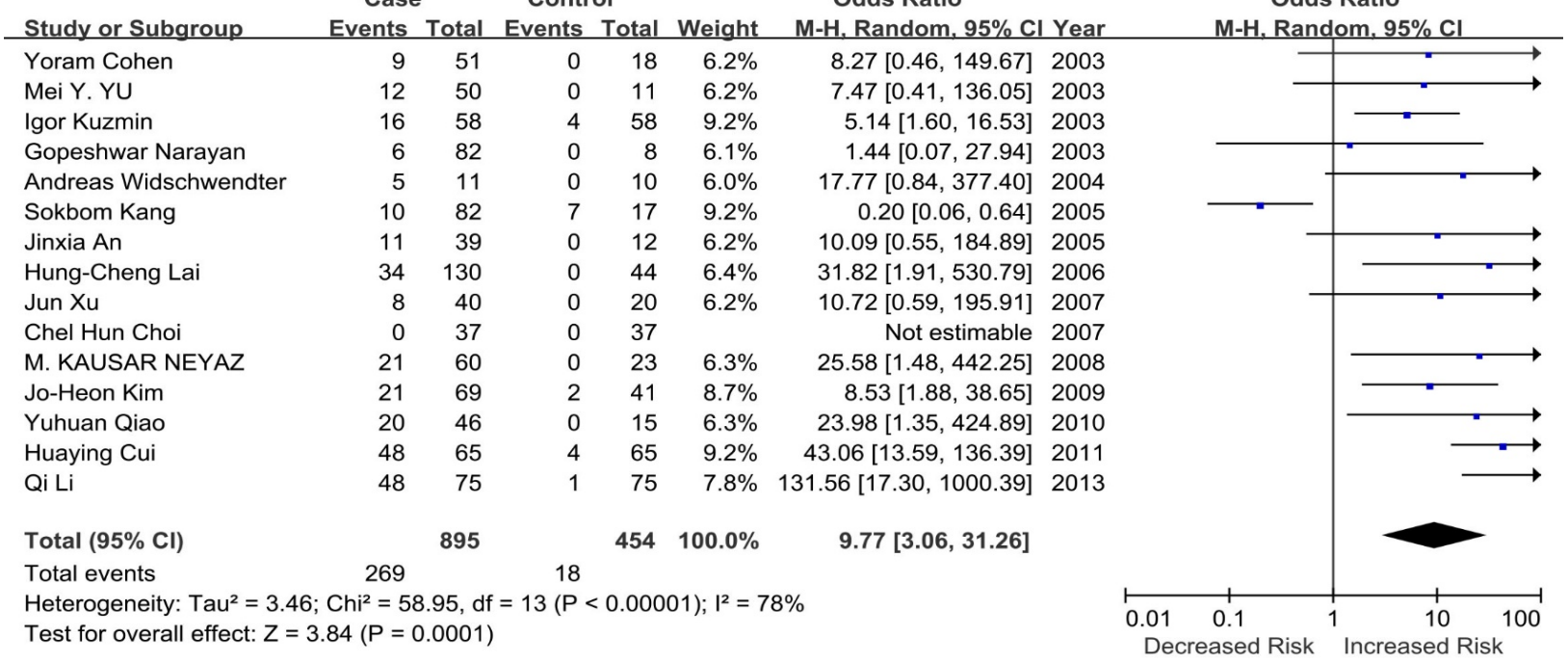

Figure 2. Meta-analysis of RASSF1A Promoter Methylation Status in Cervical Cancers 
RASSF1A Promoter Hypermethylation and Oncogenic HPV Infection in Invasive Cervical Cancer: a Meta-analysis

a total of 15 studies, representing 895 cervical cancer cases and 454 corresponding controls, for inclusion in our subsequent meta-analyses (Figure 1).

The $R A S S F 1 A$ promoter methylation frequency ranged from $0 \%$ to $73.85 \%$ (median $=26.15 \%$ ) in cervical cancer tissues and from $0 \%$ to $41.18 \%$ (median $=0 \%$ ) in the paired normal tissues. Our meta-analysis included 15 studies with an overall number of 895 cases and 454 controls and showed a statistically significant correlation between RASSF $1 A$ promoter hypermethylation and cervical cancer $\left(\mathrm{OR}=9.77,95 \% \mathrm{CI}=(3.06,31.26), P=0.0001, \mathrm{I}^{2}=78 \%\right.$, Figure 2). A further grouping of the cases within the meta- analysis by specific cervical cancer subtypes showed that aberrant RASSF 1A promoter hypermethylation tended to be more common in ACs or ASCs (median=24.37\%) than in SCCs (median=12.26\%), although the difference did not reach statistical significance $(\mathrm{OR}=0.48,95 \% \mathrm{CI}=(0.15$, 1.53), $P=0.21, \mathrm{I}^{2}=80 \%$, Figure 3 ).

As the critical etiological factor of cervical cancer, oncogenic HPV infection could be detected in most of the invasive cervical carcinomas. Among the 6 studies that included the HPV infection information, the frequency of oncogenic HPV infection ranged from $80 \%$ to $100 \%$ in the invasive SCCs (median $=80.95 \%$ ) and from $40 \%$ to $100 \%$

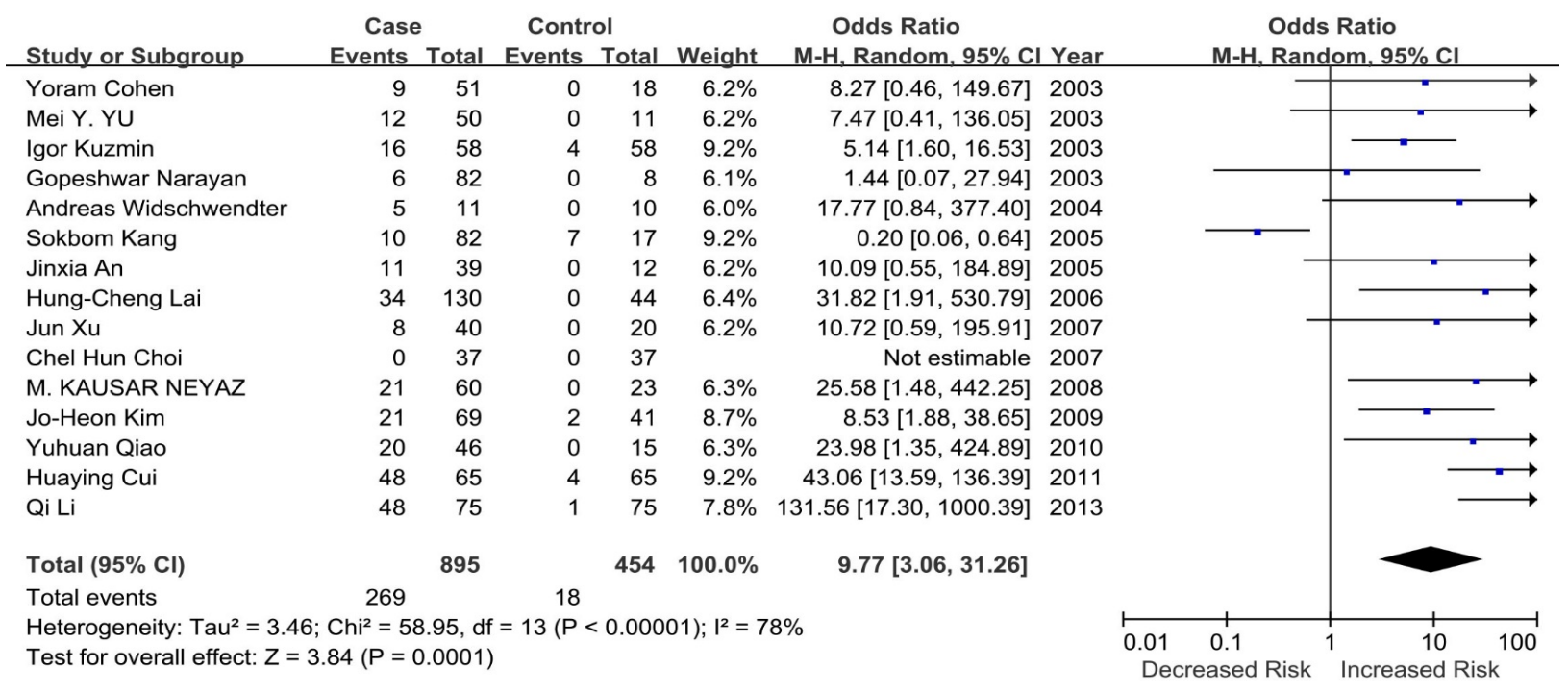

Figure 3. Meta-analysis of RASSF1A Promoter Methylation Status in Different Histological Subtypes of Cervical Cancer

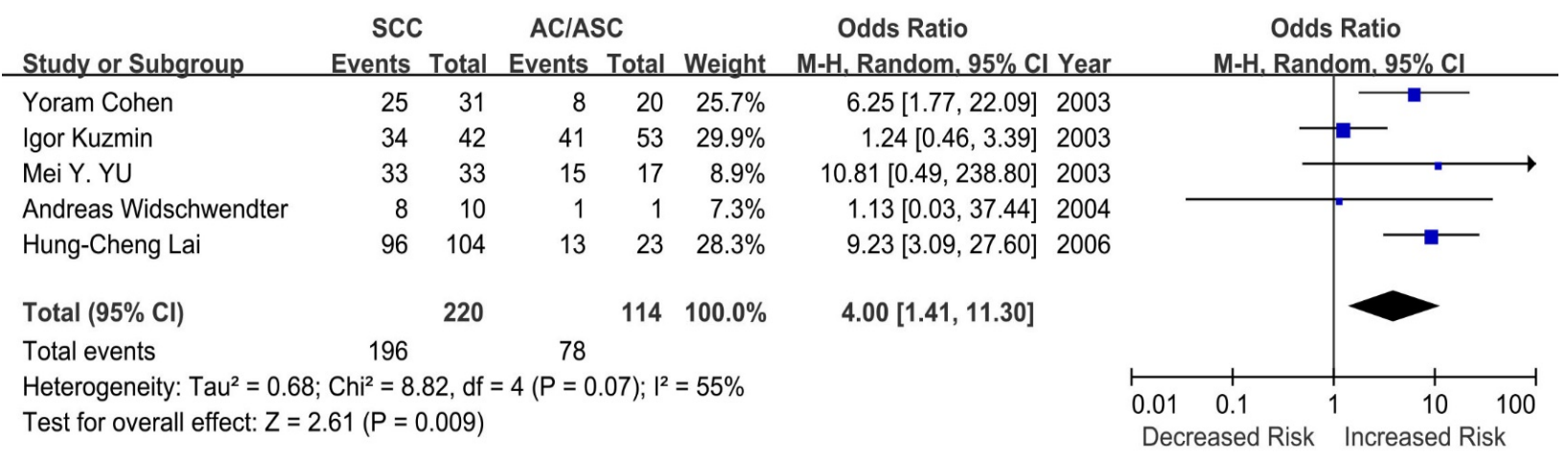

Figure 4. Subgroup Meta-analysis Segregated by HPV Infection Status in Different Histological Subtypes of Cervical Cancer

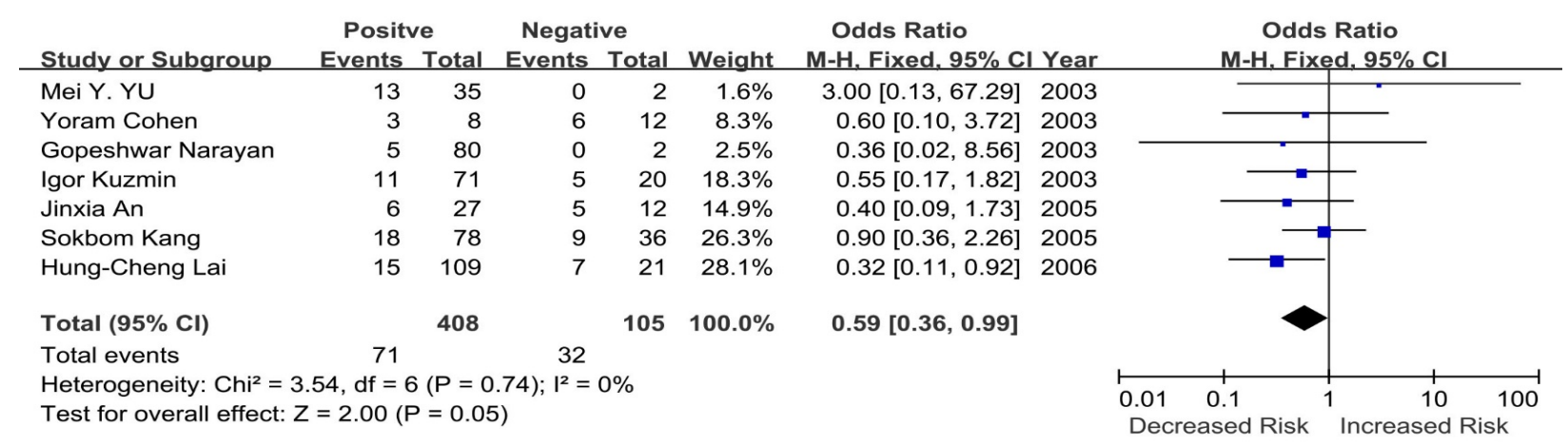

Figure 5. Meta-analysis of RASSF1A Promoter Methylation and HPV Infection Status 
Table 1. Comparison of the RASSF1A Promoter Methylation Frequency in Invasive Cervical Carcinomas

\begin{tabular}{|c|c|c|c|c|c|}
\hline \multirow[t]{2}{*}{ Author } & \multirow[t]{2}{*}{ Year } & \multicolumn{2}{|c|}{ Methylation frequency } & \multirow[t]{2}{*}{ Overall OR(95\% CI $) \mathrm{a}$} & \multirow[t]{2}{*}{$P$ value } \\
\hline & & Case & Control & & \\
\hline MeiY.YU & 2003 & $24 \%$ & $0 \%$ & $9.77[3.06,31.26]$ & 0.0001 \\
\hline Igor Kuzmin & 2003 & $27.6 \%$ & $6.9 \%$ & & \\
\hline Gopeshwar Narayan & 2003 & $7.32 \%$ & $0 \%$ & & \\
\hline Yoram Cohen & 2003 & $17.7 \%$ & $0 \%$ & & \\
\hline Andreas Widschwendter & 2004 & $45.5 \%$ & $0 \%$ & & \\
\hline Jinxia An & 2005 & $28.2 \%$ & $0 \%$ & & \\
\hline Sokbom Kang & 2005 & $12.2 \%$ & $41.2 \%$ & & \\
\hline Hung-Cheng Lai & 2006 & $26.2 \%$ & $0 \%$ & & \\
\hline Jun Xu & 2007 & $20 \%$ & $0 \%$ & & \\
\hline Chel Hun Choi & 2007 & $0 \%$ & $0 \%$ & & \\
\hline M. KAUSAR NEYAZ & 2008 & $35 \%$ & $0 \%$ & & \\
\hline Jo-Heon Kim & 2009 & $30.4 \%$ & $4.88 \%$ & & \\
\hline Yuhuan Qiao & 2010 & $0 \%$ & $0 \%$ & & \\
\hline Huaying Cui & 2011 & $73.9 \%$ & $6.15 \%$ & & \\
\hline Qi Li & 2013 & $64 \%$ & $1.33 \%$ & & \\
\hline Mean & & $26.2 \%$ & $0 \%$ & & \\
\hline
\end{tabular}

*adds ratio (OR) representing the gene methylation probability observed in cervical cancer patients compared with normal controls

Table 2. Comparison of the RASSF1A Promoter Methylation Frequency in Different Histological Subtypes of Cervical Carcinoma

\begin{tabular}{|c|c|c|c|c|c|}
\hline \multirow[t]{2}{*}{ Author } & \multirow[t]{2}{*}{ Year } & \multicolumn{2}{|c|}{ Methylation frequency } & \multirow[t]{2}{*}{ Overall OR(95\% CI $)^{\mathrm{b}}$} & \multirow[t]{2}{*}{$P$ value } \\
\hline & & $\mathrm{SCC}$ & $\mathrm{AC} / \mathrm{ASC}$ & & \\
\hline Mei Y. YU & 2003 & $30.3 \%$ & $11.8 \%$ & $0.48[0.15,1.53]$ & 0.21 \\
\hline Igor Kuzmin & 2003 & $9.52 \%$ & $22.7 \%$ & & \\
\hline Gopeshwar Narayan & 2003 & $6.49 \%$ & $20 \%$ & & \\
\hline Yoram Cohen & 2003 & $0 \%$ & $45 \%$ & & \\
\hline Hung-Cheng Lai & 2006 & $15 \%$ & $26.1 \%$ & & \\
\hline Sokbom Kang & 2007 & $1.40 \%$ & $23.5 \%$ & & \\
\hline Huaying Cui & 2011 & $82 \%$ & $46.7 \%$ & & \\
\hline Qi Li & 2013 & $64.3 \%$ & $68.4 \%$ & & \\
\hline Median & & $12.3 \%$ & $24.8 \%$ & & \\
\hline
\end{tabular}

*b Odds ratio (OR) represents the gene methylation probability observed in SCCs compared with ACs and/or ASCs

Table 3. Comparison of the HPV Infection Status in Different Histological Subtypes of Cervical Carcinoma

\begin{tabular}{lccccc}
\hline Author & Year & \multicolumn{2}{c}{ HPV infection frequency } & Overall OR(95\% CI) & $P$ value \\
\hline Mei Y. YU & & SCC & AC/ASC & & \multirow{2}{*}{0.009} \\
Igor Kuzmin & 2003 & $100 \%$ & $88.2 \%$ & $4.00[1.41,11.30]$ & \\
Yoram Cohen & 2003 & $81.0 \%$ & $77.4 \%$ & & \\
Andreas Widschwendter & 2003 & $80.7 \%$ & $40 \%$ & & \\
Hung-Cheng Lai & 2004 & $80 \%$ & $100 \%$ & & \\
Median & 2006 & $92.3 \%$ & $56.5 \%$ & & \\
\hline
\end{tabular}

* Odds ratio (OR) representing the HPV infection probability observed in SCCs compared to ACs and/or ASCs.

Table 4. Comparison of the RASSF 1A Promoter Methylation Status in Cervical Cancers with or without Detectable hrHPVs DNA

\begin{tabular}{lllccc}
\hline Author & Year & \multicolumn{2}{l}{ HPV infection frequency } & ${\text { Overall OR }(95 \% \mathrm{CI})^{\mathrm{C}}}$ & $P$ value \\
& & SCC & AC/ASC & & 0.05 \\
\hline Mei Y. YU & 2003 & $37.1 \%$ & $0 \%$ & $0.59[0.36,0.99]$ & \\
Igor Kuzmin & 2003 & $15.5 \%$ & $25 \%$ & & \\
Gopeshwar Narayan & 2003 & $6.25 \%$ & $0 \%$ & \\
Yoram Cohen & 2003 & $37.5 \%$ & $50 \%$ & \\
Jinxia An & 2005 & $22.2 \%$ & $41.7 \%$ & \\
Hung-Cheng Lai & 2006 & $13.8 \%$ & $33.3 \%$ & & \\
Sokbom Kang & 2007 & $23.1 \%$ & $25 \%$ & & \\
Median & & $22.2 \%$ & $25.0 \%$ & & \\
\end{tabular}

*d Odds ratio (OR) representing the probability of RASSF1A promoter methylation observed in HPV-positive cervical cancers compared with HPVnegative cervical cancers 
RASSF1A Promoter Hypermethylation and Oncogenic HPV Infection in Invasive Cervical Cancer: a Meta-analysis

in the ACs or ASCs (median=77.36\%). Subsequent metaanalysis suggested a significantly increased likelihood of hrHPV infection in SCCs compared with that of ACs or ASs $\left(\mathrm{OR}=4.00,95 \% \mathrm{CI}=(1.41,11.30), P=0.009, \mathrm{I}^{2}=55 \%\right.$, Figure 4).

Among the 7 studies that included both HPV infection and RASSF $1 A$ promoter methylation information, we found that $R A S S F 1 A$ hypermethylation ranged from $6.25 \%$ to $37.5 \%$ (median=22.22\%) in hrHPV-positive cervical cancer samples and from $0 \%$ to $50 \%$ (median=25\%) in hrHPV-negative samples. Interestingly, a further stratification within the meta-analysis, including 7 studies totalizing $408 \mathrm{hrHPV}$-positive cervical cancer patients and 105 hrHPV-negative patients, showed that the RASSF 1A promoter hypermethylation rate was significantly lower in hrHPV-positive patients $(\mathrm{OR}=0.59,95 \% \mathrm{CI}=(0.36$ $0.99), P=0.05, \mathrm{I}^{2}=0 \%$, Figure 5), suggesting an interactive relationship between these cervical cancer risk factors.

\section{Discussion}

Previous studies have shown that the frequency of RASSF $1 A$ promoter methylation was significantly higher in cervical cancer tissues compared with the corresponding adjacent normal tissue or with benign cervical disease tissue. In parallel, the RASSF $1 A$ expression was silenced or down-regulated in many cancer cells, including cervical cancer cells (Cohen et al., 2003; Brown et al., 2014; Feng et al., 2014), which suggested that promoter hypermethylation might lead to a reduction of RASSF $1 A$ expression.

Our meta-analysis revealed that RASSF1A promoter hypermethylation was highly associated with cervical cancer risk. An additional subgroup analysis according to different tumor histological parameters demonstrated that RASSF 1A promoter hypermethylation tended to occur more frequently in ACs/ASCs than in SCCs. The lack of statistical significance in the subgrouped meta-analysis suggested a lack of power in the comparison or that other factors could affect the development of SCC and AC/ASC.

Persistent infection by certain oncogenic HPV types was well established as the underlying cause of most premalignant and malignant epithelial lesions of the cervix (Castellsagué, 2008). The most frequent viral DNA types observed in invasive cervical carcinomas were HPVs 16, 18,45 and 31, which would account for almost $100 \%$ of the cases (Bosch et al., 1995; Walboomers et al., 1999). Interestingly, methylated RASSF $1 A$ promoter and oncogenic HPVs DNA could be detected simultaneously in ACs or ASCs, whereas in SCCs, the absence of RASSF 1A promoter methylation coupled with the high presence of HPVs DNA (Cohen et al., 2003; Kuzmin et al., 2003; Yu et al., 2003; Lai et al., 2007). In the current meta-analysis, we combined 7 related studies and identified a significant inverse correlation between RASSF $1 A$ promoter hypermethylation and the presence of oncogenic HPVs, implying that these two interactive factors might potentially determine the development of cervical cancer subtypes.

Persistent HPV infections introduce the expression of E6 and E7 papillomavirus proteins resulting in the inactivation or deregulation of $p 53$ and $p R b$ tumor suppressor genes and leading to the immortalization of primary human epithelial cells that eventually develop into cancer (Scheffner et al., 1990). The RASSF1A protein promotes apoptosis and arrests the cells in the G1 phase of the cell cycle by inhibiting the accumulation of native cyclin D1 and by engaging the Rb family-dependent cell cycle checkpoint (Shivakumar et al., 2002; Agathanggelou et al., 2005). The E7 papillomavirus protein can bypass $\mathrm{Rb}$ family-dependent cell cycle regulation by directly inhibiting the $\mathrm{Rb}$ proteins interactions (Farthing and Vousden, 1994), while the RASSF1A protein participates with the Rb family to regulate cell cycle arrest. Moreover, cancer cells expressing the $\mathrm{E} 7$ papillomavirus protein were resistant to RASSF $1 A$-induced cell cycle arrest, suggesting an interactive relationship between RASSF 1A and HPV infection. These findings might explain the interesting phenomenon identified by our results regarding the interaction between these risk factors. However, further studies are needed to clarify the interactive mechanisms by which the RASSF1A protein and HPV contribute to the development of specific cervical cancer subtypes.

Although our meta-analysis was performed with careful screening of numerous relevant studies, several limitations must be considered. Above all, conference abstracts and inaccessible full-text articles were excluded from our meta-analysis because we were unable to retrieve the relevant data for the meta-analysis. Moreover, only the literature available in the English or Chinese languages was selected, neglecting results reported in other languages, which introduces a bias in the literature selection. In addition, only 15 studies were included in our analysis. Future studies with a larger sample size are required to confirm our findings.

In summary, our meta-analysis suggested that abnormal RASSF $1 A$ promoter methylation and HPV infection, identified as two seemingly separate etiological factors in cervical carcinomas, might actually act competitively by involving similar cellular signal transduction pathways. However, the precise mechanistic connection between HPV infection and RASSF $1 A$ inactivation remains to be determined.

\section{Acknowledgement}

The research was supported by the grants from National Natural Science Foundation of China (31100919 and 81371469), Natural Science Foundation of Zhejiang Province (LR13H020003), K. C. Wong Magna Fund in Ningbo University, and Ningbo Natural Science Foundation (2014A610235). The funders had no role in study design, data collection and analysis, decision to publish, or preparation of the manuscript.

\section{References}

Agathanggelou A, Cooper WN, Latif F (2005). Role of the Ras-association domain family 1 tumor suppressor gene in human cancers. Cancer Res, 65, 3497-508.

Bird AP (1986). CpG-rich islands and the function of DNA methylation. Nature, 321, 209-13. 


\section{Jin-Yun Li et al}

Bosch FX, Manos MM, Munoz N, et al (1995). Prevalence of human papillomavirus in cervical cancer: a worldwide perspective. International biological study on cervical cancer (IBSCC) Study Group. J Natl Cancer Inst, 87, 796-802.

Brown TC, Juhlin CC, Healy JM, et al (2014). Frequent silencing of RASSF $1 A$ via promoter methylation in follicular thyroid hyperplasia: a potential early epigenetic susceptibility event in thyroid carcinogenesis. JAMA Surg.

Castellsagué X (2008). Natural history and epidemiology of HPV infection and cervical cancer. Gynecol Oncol, 110, 4-7.

Cheung TH, Lo KW, Yim SF, et al (2004). Epigenetic and genetic alternation of PTEN in cervical neoplasm. Gynecol Oncol, 93, 621-7.

Cohen Y, Singer G, Lavie O, et al (2003). The RASSF1A tumor suppressor gene is commonly inactivated in adenocarcinoma of the uterine cervix. Clin Cancer Res, 9, 2981-4.

Farthing AJ, Vousden KH (1994). Functions of human papillomavirus E6 and E7 oncoproteins. Trends Microbiol, 2, 170-4.

Feng L, Li J, Yan LD, et al (2014). RASSF1A suppresses proliferation of cervical cancer cells. Asian Pac J Cancer Prev, 15, 5917-20.

Jha AK, Nikbakht M, Jain V, et al (2012). p16(INK4a) and p15(INK4b) gene promoter methylation in cervical cancer patients. Oncol Lett, 3, 1331-5.

Jiang D, Hong Q, Shen Y, et al (2014a). The diagnostic value of DNA methylation in leukemia: a systematic review and meta-analysis. PLoS One, 9, 96822.

Jiang D, Shen Y, Dai D, et al (2014b). Meta-analyses of methylation markers for prostate cancer. Tumour Biol.

Kuzmin I, Liu L, Dammann R, et al (2003). Inactivation of RAS association domain family $1 \mathrm{~A}$ gene in cervical carcinomas and the role of human papillomavirus infection. Cancer Res, 63, 1888-93.

Lai HC, Lin YW, Chang CC, et al (2007). Hypermethylation of two consecutive tumor suppressor genes, BLU and RASSF 1A, located at 3p21.3 in cervical neoplasias. Gynecol Oncol, 104, 629-35.

Lipsey MW, Wilson DB 2001. Practical meta-analysis, Thousand Oaks, Calif., Sage Publications.

Maliukova AV, Loginov VI, Khodyrev DS, et al (2004). (Methylation of the putative tumor suppressor gene, RASSF1A, in primary cervical tumors). Mol Biol (Mosk), 38, 1005-13.

Munoz N, Bosch FX, de Sanjose S, et al (2003). Epidemiologic classification of human papillomavirus types associated with cervical cancer. $N$ Engl J Med, 348, 518-27.

Richter AM, Pfeifer GP, Dammann RH (2009). The RASSF proteins in cancer; from epigenetic silencing to functional characterization. Biochim Biophys Acta, 1796, 114-28.

Scheffner M, Werness BA, Huibregtse JM, et al (1990). The E6 oncoprotein encoded by human papillomavirus types 16 and 18 promotes the degradation of $\mathrm{p} 53$. Cell, 63, 1129-36.

Shivakumar L, Minna J, Sakamaki T, et al (2002). The RASSF1A tumor suppressor blocks cell cycle progression and inhibits cyclin D1 accumulation. Mol Cell Biol, 22, 4309-18.

van der Weyden L, Adams DJ (2007). The Ras-association domain family (RASSF) members and their role in human tumourigenesis. Biochim Biophys Acta, 1776, 58-85.

Walboomers JM, Jacobs MV, Manos MM, et al (1999). Human papillomavirus is a necessary cause of invasive cervical cancer worldwide. J Pathol, 189, 12-9.

Yu MY, Tong JH, Chan PK, et al (2003). Hypermethylation of the tumor suppressor gene RASSFIA and frequent concomitant loss of heterozygosity at $3 \mathrm{p} 21$ in cervical cancers. Int $J$ Cancer, 105, 204-9.

Zhu X, He F, Zeng H, et al (2014). Identification of functional cooperative mutations of SETD2 in human acute leukemia. Nat Genet, 46, 287-93. 